Chronic Obstructive Pulmonary Diseases: Journal of the COPD Foundation

\title{
Perspective
}

\section{MicroRNAs as Therapeutic Targets in Lung Disease: Prospects and Challenges}

\author{
Tadashi Sato, MD, $\mathrm{PhD}^{1}$ Hario Baskoro, $\mathrm{MD}^{1}$ Stephen I. Rennard, $\mathrm{MD}^{2,3}$ Kuniaki Seyama, MD, $\mathrm{PhD}^{1}$ \\ Kazuhisa Takahashi, MD, $\mathrm{PhD}^{1}$
}

\begin{abstract}
MicroRNAs (miRNAs) are small non-coding RNAs that regulate multiple target genes providing fine-tuned coordinated expression. Growing evidence suggests that miRNAs play important roles in lung development and the pathogenesis of lung disease and that they have great potential as novel therapeutic targets for the treatment of diseases such as lung cancer, asthma, pulmonary fibrosis, and chronic obstructive pulmonary disease (COPD). We have previously shown that miR-146a is a promising therapeutic target for controlling abnormal inflammatory response in COPD through a series of in vitro experiments in lung fibroblasts. However, further investigations in in vivo experimental models are needed to explore the role of miR-146a in the pathogenesis and therapy of COPD. Recently, miRNAs encapsulated in extracellular vesicles (EVs) have been recognized as modulators of intercellular communication. EVs, therefore, may also have therapeutic potential and show promise for use as biomarkers for various lung diseases. In addition to miRNAs, we briefly discuss a specific long non-coding RNA (lncRNA) that may contribute to the pathogenesis of COPD. The application of miRNA-based therapeutics faces several challenges related to mode of delivery, stability, and tissue specificity. However, recent advances in nanotechnology are expected to prove valuable for the development of miRNA-based therapeutics to treat lung disease.
\end{abstract}

\begin{abstract}
Abbreviations: microRNA, miRNA; chronic obstructive pulmonary disease, COPD; extracellular vesicles, EVs; long non-coding RNA, lncRNA; RNA-induced silencing complex, RISC; T helper type 2, Th2; interleukin, IL; tumor necrosis factor, TNF; prostaglandin $\mathrm{E}_{2}$, PGE $\mathbf{2}_{\mathbf{2}}$; cyclooxygenase-2, COX-2; nuclear factor, NF; cigarette smoke extract, CSE; the smoke and cancer-associated lncRNA-1, SCAL-1; nuclear factor erythroid 2-related factor, NRF2

Funding Support: This work was supported by Grant-in-Aid for Scientific Research (T.S., 26461199) and the Institute for Environmental and Gender-Specific Medicine, Juntendo University Graduate School of Medicine (T.S.).

Date of Acceptance: October 15, 2015

Citation: Sato T, Baskoro H, Rennard SI, Seyama K, Takahashi K. MicroRNAs as therapeutic targets in lung disease: Prospects and challenges. Chronic Obstr Pulm Dis (Miami). 2016; 3(1):382-388. doi: http://dx.doi.org/10.12653/jcopdf.2015.3.1.0160
\end{abstract}

1 Department of Respiratory Medicine, Juntendo University Graduate School of Medicine, Tokyo, Japan

2 Pulmonary, Critical Care, Sleep and Allergy Medicine, Department of Internal Medicine, University of Nebraska Medical Center, Omaha

3 Clinical Discovery Unit, AstraZeneca, Cambridge, United Kingdom

\section{Address correspondence to:}

Tadashi Sato, MD, PhD

Phone: 81-3-5802-1063

Fax: 81-3-5802-1617

E-mail: satotada@juntendo.ac.jp

\section{Keywords:}

microRNA; lung diseases; chronic obstructive pulmonary disease; miRNA-based therapeutics; extracellular vesicles; long non-coding RNA

\section{Introduction}

MicroRNAs (miRNAs) have been the focus of much attention worldwide since their discovery by Ambros et al in 1993. ${ }^{1}$ Lin-4 was the first miRNA to be identified through a study of developmental timing in the nematode Caenorhabditis elegans. miRNAs 
are non-coding endogenous RNAs, approximately 19- to 25-nucleotides long, which play a role in the post-transcriptional regulation of gene expression. Briefly, miRNA biogenesis begins with the cleavage of a pri-miRNA into a pre-miRNA, which is mediated by the Drosha enzyme (Figure 1). The pre-miRNA is then actively transported from the nucleus into the

\section{Figure 1. MicroRNA Biogenesis}

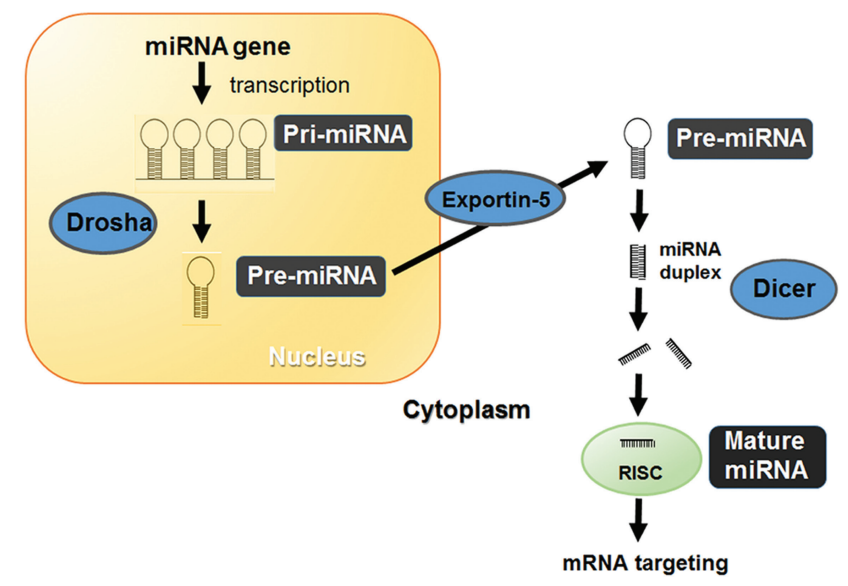

miRNA biogenesis begins with the cleavage of pri-miRNA into pre-miRNA by the Drosha enzyme. The pre-miRNA is transported from the nucleus into the cytoplasm by Exportin-5. Dicer processes the pre-miRNA to generate a double-stranded RNA, of which 1 strand is degraded and the other is incorporated into RISC, which functions as a mature miRNA against its target mRNAs.

cytoplasm by Exportin-5. Next, the RNase III enzyme Dicer processes the pre-miRNA to generate a doublestranded RNA. One strand is degraded and the other is incorporated into the RNA-induced silencing complex (RISC), which then functions as a mature miRNA against its target mRNAs. The mature miRNAs, which are expressed during development in a tissue- or cell typespecific manner, mediate post-transcriptional repression of gene expression by increasing mRNA degradation or by inhibiting translation. ${ }^{2}$ This is achieved by direct binding between the miRNA 5' region and the 3' untranslated region of the target mRNA. miRNAs may also indirectly alter gene expression by epigenetic mechanisms such as DNA methylation and histone acetylation. ${ }^{3,4}$ These processes enable a fine tuning of gene expression as opposed to an on-off switch. ${ }^{5}$ To date, over 4000 miRNAs have been discovered in multicellular eukaryotes and more than 1000 miRNAs have been identified in humans. MiRNAs have been found to be associated with diverse biological processes such as development, cellular differentiation, and the pathogenesis of various diseases.

\section{MicroRNAs Roles in Lung Development and Disease}

The various roles of miRNAs in lung development and disease have been studied widely (shown in Table 1). miR-17-92-deficient mice were found to possess underdeveloped lungs, whereas mice in which miR-

\section{Table 1. Representative MicroRNAs Associated with Lung Development and Disease}

\begin{tabular}{l|r|r}
\hline Disease/Condition & MicroRNA(s) & Reference(s) \\
\hline Lung Development & miR-17-92 & 6,7 \\
\hline Immune Response & miR-125b, 132, 146a, 155 & 8,9 \\
\hline Lung Cancer & let-7, miR-17-92 & $10,11,25,26$ \\
\hline Asthma & miR-126, 133a, 155 & $12,13,14,15$ \\
\hline Pulmonary Fibrosis & let-7d, miR-21 & 16,17 \\
\hline COPD & miR-218 & 18 \\
\hline & miR-15b & 19 \\
\hline miR-146a & 20 \\
\hline & miR-1, 499, 133, 206 & 25,26 \\
\hline let-7c & 27 \\
\hline miR-101, 144 & 29 \\
\hline miR-638 & 30 \\
\hline
\end{tabular}

17-92 was overexpressed showed highly proliferative undifferentiated epithelia, ${ }^{6,7}$ indicating that this miRNA plays a role in lung development. miRNAs also act as immunomodulators, as indicated by the upregulation of miR-132, miR-146a, and miR-155 and downregulation of miR-125b in macrophage cell lines in response to stimulation with endotoxin. 8,9 The pathogenetic roles of miRNAs in lung diseases such as lung cancer, asthma, pulmonary fibrosis, and chronic obstructive pulmonary disease (COPD) have also been investigated. There have already been numerous reports that focus on miRNAs in lung cancer. For example, the Let-7 gene is known for its role as a tumor suppressor, whereas miR17-92 functions as an oncogene in lung cancer. ${ }^{10,11}$ To date, in vitro and in vivo studies have implicated several miRNAs as crucial factors for the pathogenesis of asthma. Mattes et al showed that $\mathrm{T}$ helper type 2 (Th2)-associated inflammation induces increased expression of miR-126 and selective blockade of miR126 suppresses the asthmatic phenotype. ${ }^{12}$ On the other hand, Chiba et al reported decreased expression of miR-133a in bronchial smooth muscle cells following 
treatment with a major Th2 cytokine, interleukin (IL)-13. ${ }^{13}$ In addition, Rodriguez et al reported that miR-155 knockout mice developed an asthma-like phenotype with inflammatory infiltration into the lung and spontaneous airway remodeling. ${ }^{14}$ Our group also reported that miR-155 is associated with the asthmatic phenotype of fibroblast-like cells derived from human embryonic stem cells. ${ }^{15}$ We have demonstrated that IL-4 induces differentiation of stem/precursor cells into fibroblast-like cells that demonstrate a more fibrogenic phenotype, which is due to reduced expression of miR155. Regarding pulmonary fibrosis, Liu et al reported that the expression of miR-2 1 is increased in both a murine fibrosis model and in human specimens. ${ }^{16}$ Furthermore, Pandit et al reported that the downregulation of let- $7 \mathrm{~d}$ expression by administration of antagomir resulted in increased epithelial-mesenchymal transition changes in vitro and collagen deposition and alveolar septal thickening in vivo. ${ }^{17}$

Cigarette smoking is the main cause of COPD; however, the pathogenetic mechanisms underlying this disease are poorly understood. Epigenetic regulation is considered to play an important role in the pathogenesis of COPD. Schembri et al examined whole-genome miRNA and mRNA expression in the bronchial airway epithelium in current and never smokers and found that miR-218 levels modulate airway epithelial gene expression in response to exposure to cigarette smoke. ${ }^{18}$ Ezzie et al compared the miRNA profile in lung tissue from non-COPD smokers with that in lung tissue from COPD individuals and found that 70 miRNAs were differentially expressed and that miR-15b, in particular, was increased in COPD. ${ }^{19}$ miR-15b was shown to be increased in COPD samples compared with controls and was differentially expressed in correlation with disease severity by the Global initiative for obstructive Lung Disease (GOLD) classification. The authors have further described that expression of Smad7, which was validated as a target for miR-15b, was decreased in bronchial epithelial cells in COPD. Our group reported reduced expression of miR-146a in lung fibroblasts from COPD patients compared with that observed in lung fibroblasts from non-COPD patients, following stimulation by the inflammatory cytokines, IL-1 $\beta$ and tumor necrosis factor (TNF)- $\alpha^{20}$ As shown in Figure 2, we further showed that miR-146a causes downregulation of the production of prostaglandin $\mathrm{E}_{2}\left(\mathrm{PGE}_{2}\right)$, an inflammatory mediator, by targeting cyclooxygenase-2 (COX-2). Interestingly, miR-146a

\section{Figure 2. Differences in Response to Inflammatory Cytokines: Control Fibroblasts Versus COPD Fibroblasts}

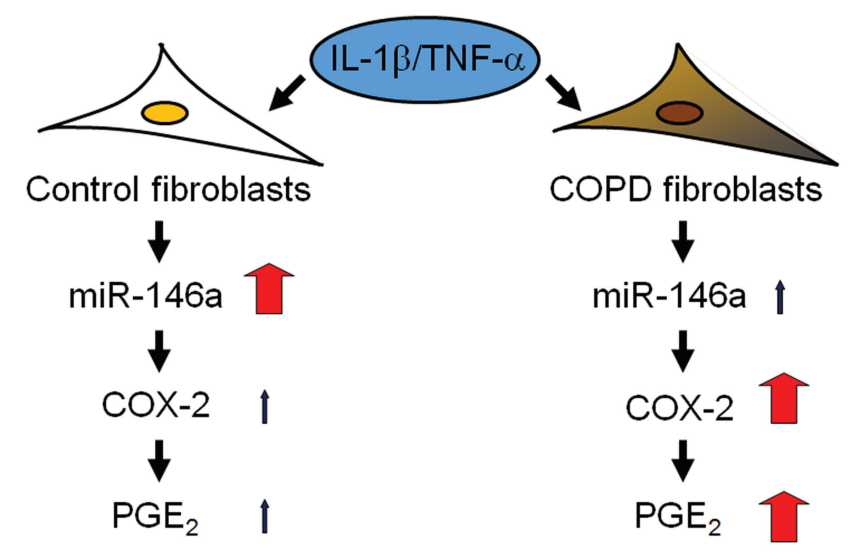

Schema showing the differences in response to inflammatory cytokines between control fibroblasts and fibroblasts from COPD patients (COPD fibroblasts). Fibroblasts were isolated from parenchymal tissue obtained from smokers, with and without COPD, undergoing lung surgery. Following IL- $1 \beta$ and TNF- $\alpha$ stimulation, control (without COPD) fibroblasts were found to induce sufficient expression of miR-146a for the regulation of COX-2 expression and $\mathrm{PGE}_{2}$ production, whereas COPD fibroblasts were unable to induce sufficient expression of miR-146a, resulting in the overexpression of COX-2 and $\mathrm{PGE}_{2}$.

levels were found to be increased in synovial tissue from individuals with rheumatoid arthritis, suggesting that this miRNA is also associated with the pathogenetic mechanisms underlying other inflammatory diseases. ${ }^{21,22}$ miR-146a is also known to downregulate nuclear factor (NF)- $\mathrm{kB}$ activity by repressing the NF- $\mathrm{kB}$ transducers IL-1 receptor-associated kinase and TNF receptor-associated factor $6 .^{8}$ We, therefore, concluded that miR-146a may represent a promising therapeutic target for controlling abnormal inflammatory response in COPD. ${ }^{20}$ However, further investigations in in vivo experimental models are needed to explore the role of miR-146a in the pathogenesis and therapy of COPD.

Other miRNAs have also been reported to be associated with the pathogenesis of COPD. ${ }^{23,24}$ Lewis et al described that miR-1, which is one of the musclespecific miRNAs, is downregulated in the quadriceps of patients with COPD. ${ }^{25}$ The same group also showed that skeletal muscle-specific miRNAs (miR-1, 499, 133 and 206) are elevated in the plasma of stable COPD compared with controls. ${ }^{26}$ The authors speculated that these miRNAs may contribute to the development of skeletal muscle dysfunction in COPD and may be a future diagnostic tool for that condition. Van 
Pottelberge et al analyzed the miRNA profile in induced sputum and showed that let-7c is significantly reduced in COPD individuals compared with smokers without COPD. $^{27}$ Moreover, let-7c has been associated with increased expression of TNF receptor II, which may be related to the pathogenesis of COPD as demonstrated by a TNF receptor II knockout mice study. ${ }^{27,28}$ Hassan et al examined the effect of cigarette smoke extract (CSE) on the human bronchial epithelial cells and showed that miR-101 and miR-144 are upregulated by CSE and suppress the cystic fibrosis transmembrane conductance regulator protein, a chloride channel involved in the maintenance of fluid homeostasis in the lung. ${ }^{29}$ A recent analysis of human lung tissue with regional emphysema revealed that miR-638 is positively correlated with emphysema severity and regulates gene expression networks associated with the oxidative stress response and aging in emphysematous lung tissue. ${ }^{30}$

\section{MicroRNAs Novel Therapeutic Targets}

MiRNAs show great potential as novel therapeutic targets for the treatment of numerous diseases. To date, several miRNAs have been developed as drugs and tested in clinical trials. For example, Santaris Pharma has developed a locked nucleic acid-based antisense oligonucleotide that is targeted to the liver following intravenous injection, where it effectively inhibits miR122 , thereby playing a positive role in the regulation of hepatitis C viral replication. ${ }^{31}$ This oligonucleotide has already passed through Phase II clinical trials with promising results in patients with HCV infection. ${ }^{32}$ In the field of respiratory medicine, attempts are underway to develop a let-7 mimetic-based therapeutic agent for lung cancer; however, this candidate is currently still at the pre-clinical trial stage. ${ }^{33,34}$

The application of miRNA-based therapeutics is limited by several challenges related to mode of delivery, stability, and tissue specificity of the therapeutic agent. ${ }^{35}$ As the lung is accessible to therapeutic agents via the intrapulmonary route, aerosolization is a strategy for enhancing drug delivery and reducing side effects. Free miRNAs are rapidly degraded by nucleases in serum and extracellular fluids. Chemical modification at specific positions or formulation with delivery vectors have been shown to improve stability; however, these strategies may attenuate the suppressive activity of the oligonucleotides. ${ }^{36}$ Fujita et al recently developed a novel platform to enable naked RNA-interference approaches involving administration via inhalation in mice, without the need for sophisticated delivery technology. ${ }^{37}$ Additionally, anti-miRNA therapy utilizing chemically modified anti-miR oligonucleotides is reported to show potential as a therapy for lung disease, as the oligonucleotides can be successfully delivered without the requirement of delivery vectors. ${ }^{38}$ More recently, growing evidence suggests that EVs such as exosomes and microvesicles play pathogenetic roles in various lung diseases. ${ }^{39} \mathrm{EVs}$, which are released from respiratory cells and immune cells, encapsulate various molecules including proteins and miRNAs and act as modulators of intercellular communication. Recent evidence indicates that the various respiratory cellderived EVs play a key role for maintaining physiological homeostasis. Ismail et al reported that miR-223 contained in macrophage-derived EVs was transported to target cells, including monocytes, endothelial cells, epithelial cells and fibroblasts, resulting in cellular effects. ${ }^{40}$ In addition, Kulshreshtha et al reported that epithelial cell-derived EVs can enhance proliferation and chemotaxis of macrophages under the influence of IL$13{ }^{41}$ In addition to their possible roles in pathogenesis, EVs may have therapeutic potential and show promise for use as biomarkers for lung diseases. ${ }^{42}$ Currently, circulating endothelial cell-derived EVs have shown promise as novel biomarkers that relate to the degree of lung endothelial injury in COPD. ${ }^{43}$ Gordon et al have reported that circulating endothelial cell-derived EVs are increased in smokers compared with non-smokers. ${ }^{44}$ Takahashi et al showed that the elevation of a specific fraction of endothelial cell-derived EVs are collated with the decline of annual forced expiratory volume in 1 second through a prospective study of stable COPD patients. $^{45}$

\section{Beyond MicroRNAs-IncRNAs in Lung Disease}

Besides miRNAs, lncRNAs are also receiving attention as regulators of gene expression. LncRNAs are nonprotein coding transcripts longer than 200 nucleotides. In general, lncRNAs lack strong conservation across species, in contrast to many small RNAs such as miRNAs, and were originally classified as nonfunctional. ${ }^{46}$ The biological function of most lncRNAs remains unknown, however growing evidence suggests 
that lncRNAs play complex roles in gene regulation ${ }^{47}$ and that some lncRNAs contribute to the development of neoplasms including lung cancer. ${ }^{48,49}$ Recently, Thai et al have characterized a novel lncRNA, the smoke and cancer-associated lncRNA-1 (SCAL 1) that is induced by cigarette smoke and elevated in lung cancer cell lines. ${ }^{50}$ They also revealed that SCAL1 may act downstream of nuclear factor erythroid 2-related factor (NRF2) to regulate gene expression and protect against oxidative stress. NRF2 has been reported to play an important role in the development of smoke-induced emphysema: NRF2 knockout mice are extremely susceptible to cigarette smoke-induced emphysema. ${ }^{51}$ SCAL1, therefore, may contribute to the pathogenesis of COPD and may be a novel therapeutic target controlling oxidative stress induced by cigarette smoke exposure.

\section{Conclusion}

Emerging evidence suggests that miRNA-based approaches provide a promising therapeutic strategy for lung diseases. Since miRNAs can target multiple genes leading to a fine-tuned coordinated regulation of complex biological processes, miRNA-based therapeutics are believed to have great potential for pathologically complex diseases including COPD. Moreover, the lung is accessible to therapeutic agents via the intrapulmonary route and aerosolization may be useful for enhancing miRNA delivery and reducing side effects. While additional research will be needed to address the technical obstacles that need to be overcome in order to use miRNAs clinically, advances in nanotechnology and chemistry of RNA analogues are expected to enable the development of miRNA-based therapeutics for the treatment of various lung diseases.

\section{Acknowledgements}

The authors thank Aki Mitsui for excellent technical support.

\section{Declaration of Interest}

TS has received grants from Kyorin, Otsuka and MiZ Company. SIR has received reimbursement for attending a symposium, speaking and/or consulting from: ABIM, Able Associates, Advantage Healthcare, Align2Action, Almirall, APT, ATS, AstraZeneca, Baxter, BoehringerIngelheim, Chiesi, CIPLA, ClearView Healthcare, Cleveland Clinic, CME Incite, Complete Medical Group, COPD Foundation, Cory Paeth, CSA, CSL, CTS Carmel, Dailchi Sankyo, Decision Resources, Dunn Group, Easton Associates, Elevation Pharma, FirstWord, Forest, Frankel Group, Gerson, GlaxoSmithKline, Gilead, Grifols, GroupH, Guidepoint Global, Haymarket, HealthStar, Huron Consulting, Incite, Inthought, IntraMed (Forest), Johnson \& Johnson, LEK, McKinsey, Medical Knowledge, Mediummune, Methodist Health System (Dallas), Navigant, NCI Consulting, Novartis, Nuvis, Pearl, Penn Technology, Pfizer, PlanningShop, Prescott, Pro Ed Comm, ProiMed, PSL FirstWord, Pulmatrix, Quadrant, Qwessential, Regeneron, Saatchi and Saatchi, Schlesinger Associates, Strategic North, Synapse, Takeda, Theron, WebMD. SIR's institution has received grant support from the National Heart Lung and Blood Institute, Nebraska Department of Health and Human Services, Otsuka, Pfizer, GlaxoSmithKline, Boehringer Ingelheim, Nycomed, AstraZeneca, Centocor, Almirall. SIR is currently an employee of AstraZeneca. No other authors have conflicts of interest to declare. 


\section{References}

1. Lee RC, Feinbaum RL, Ambros V. The C. elegans heterochronic gene lin-4 encodes small RNAs with antisense complementarity to lin-14. Cell. 1993. 75(5): 843-854.

2. Nana-Sinkam SP, Hunter MG, Nuovo GJ, et al. Integrating the MicroRNome into the study of lung disease. Am J Respir Crit Care Med. 2009. 179(1): 4-10. doi: http://dx.doi.org/10.1164/rccm.200807-1042PP

3. Fabbri M, Garzon R, Cimmino A, et al. MicroRNA-29 family reverts aberrant methylation in lung cancer by targeting DNA methyltransferases 3A and 3B. Proc Natl Acad Sci U S A. 2007. 104(40): 15805-15810. doi: http://dx.doi.org/10.1073/pnas.0707628104

4. Garzon R, Liu S, Fabbri M, et al. MicroRNA-29b induces global DNA hypomethylation and tumor suppressor gene reexpression in acute myeloid leukemia by targeting directly DNMT3A and 3B and indirectly DNMT1. Blood. 2009. 113(25): 6411-6418. doi: http://dx.doi.org/10.1182/blood-2008-07-170589

5. Baek D, Villen J, Shin C, et al. The impact of microRNAs on protein output. Nature. 2008. 455(7209): 64-71. doi: http://dx.doi.org/10.1038/nature07242

6. Ventura A, Young AG, Winslow MM, et al. Targeted deletion reveals essential and overlapping functions of the miR-17 through 92 family of miRNA clusters. Cell. 2008. 132(5): 875-886. doi: http://dx.doi.org/10.1016/j.cell.2008.02.019

7. Lu Y, Thomson JM, Wong HY, Hammond SM, Hogan BL. Transgenic over-expression of the microRNA miR-17-92 cluster promotes proliferation and inhibits differentiation of lung epithelial progenitor cells. Dev Biol. 2007. 310(2): 442-453. doi: http://dx.doi.org/10.1016/j.ydbio.2007.08.007

8. Taganov KD, Boldin MP, Chang KJ, Baltimore D. NFkappaB-dependent induction of microRNA miR-146, an inhibitor targeted to signaling proteins of innate immune responses. Proc Natl Acad Sci U S A. 2006. 103(33): 1248112486. doi: http://dx.doi.org/10.1073/pnas.0605298103

9. Tili E, Michaille JJ, Calin GA. Expression and function of microRNAs in immune cells during normal or disease state. Int J Med Sci. 2008. 5(2): 73-79. doi: http://dx.doi.org/10.7150/ijms.5.73

10. Takamizawa J, Konishi H, Yanagisawa K, et al. Reduced expression of the let-7 microRNAs in human lung cancers in association with shortened postoperative survival. Cancer Res. 2004. 64(11): 3753-3756.

doi: http://dx.doi.org/10.1158/0008-5472.CAN-04-0637

11. Hayashita Y, Osada H, Tatematsu Y, et al. A polycistronic microRNA cluster, miR-17-92, is overexpressed in human lung cancers and enhances cell proliferation. Cancer Res. 2005. 65(21): 9628-9632.

doi: http://dx.doi.org/10.1158/0008-5472.CAN-05-2352

12. Mattes J, Collison A, Plank M, Phipps S, Foster PS. Antagonism of microRNA-126 suppresses the effector function of TH2 cells and the development of allergic airways disease. Proc Natl Acad Sci U S A. 2009. 106(44): 18704-18709. doi: http://dx.doi.org/10.1073/pnas.0905063106
13. Chiba Y, Tanabe M, Goto K, Sakai H, Misawa M. Down-regulation of miR-133a contributes to up-regulation of Rhoa in bronchial smooth muscle cells. Am J Respir Crit Care Med. 2009. 180(8): 713-719. doi: http://dx.doi.org/10.1164/rccm.200903-0325OC

14. Rodriguez A, Vigorito E, Clare S, et al. Requirement of bic/ microRNA-155 for normal immune function. Science. 2007. 316(5824): 608-611.

doi: http://dx.doi.org/10.1126/science.1139253

15. Sato T, Liu X, Basma H, et al. IL-4 induces differentiation of human embryonic stem cells into fibrogenic fibroblast-like cells. J Allergy Clin Immunol. 2011. 127(6): 1595-603 e9. doi: http://dx.doi.org/10.1016/j.jaci.2011.01.049

16. Liu G, Friggeri A, Yang Y, et al. miR-21 mediates fibrogenic activation of pulmonary fibroblasts and lung fibrosis. J Exp Med. 2010. 207(8): 1589-1597. doi: http://dx.doi.org/10.1084/jem.20100035

17. Pandit KV, Corcoran D, Yousef H, et al. Inhibition and role of let7d in idiopathic pulmonary fibrosis. Am J Respir Crit Care Med. 2010. 182(2): 220-229. doi: http://dx.doi.org/10.1164/rccm.200911-16980C

18. Schembri F, Sridhar S, Perdomo C, et al. MicroRNAs as modulators of smoking-induced gene expression changes in human airway epithelium. Proc Natl Acad Sci U S A. 2009. 106(7): 2319-2324. doi: http://dx.doi.org/10.1073/pnas.0806383106

19. Ezzie ME, Crawford M, Cho JH, et al. Gene expression networks in COPD: microRNA and mRNA regulation. Thorax. 2012. 67(2): 122-131. doi: http://dx.doi.org/10.1136/thoraxjnl-2011-200089

20. Sato T, Liu X, Nelson A, et al. Reduced miR-146a increases prostaglandin $\mathrm{E}(2)$ in chronic obstructive pulmonary disease fibroblasts. Am J Respir Crit Care Med. 2010. 182(8): 1020-1029. doi: http://dx.doi.org/10.1164/rccm.201001-0055OC

21. Stanczyk J, Pedrioli DM, Brentano F, et al. Altered expression of MicroRNA in synovial fibroblasts and synovial tissue in rheumatoid arthritis. Arthritis Rheum. 2008. 58(4): 1001-1009. doi: http://dx.doi.org/10.1002/art.23386

22. Nakasa T, Miyaki S, Okubo A, et al. Expression of microRNA-146 in rheumatoid arthritis synovial tissue. Arthritis Rheum. 2008. 58(5): 1284-1292. doi: http://dx.doi.org/10.1002/art.23429

23. Osei ET, Florez-Sampedro L, Timens W, et al. Unravelling the complexity of COPD by microRNAs: it's a small world after all. Eur Respir J. 2015. 46(3): 807-818. doi: http://dx.doi.org/10.1183/13993003.02139-2014

24. Booton R, Lindsay MA. Emerging role of MicroRNAs and long noncoding RNAs in respiratory disease. Chest. 2014. 146(1): 193204. doi: http://dx.doi.org/10.1378/chest.13-2736

25. Lewis A, Riddoch-Contreras J, Natanek SA, et al. Down regulation of the serum response factor/miR-1 axis in the quadriceps of patients with COPD. Thorax. 2012. 67(1): 26-34. doi: http://dx.doi.org/10.1136/thoraxjnl-2011-200309

26. Donaldson A, Natanek SA, Lewis A, et al. Increased skeletal muscle-specific microRNA in the blood of patients with COPD. Thorax. 2013. 68(12): 1140-1149. doi: http://dx.doi.org/10.1136/thoraxjnl-2012-203129 
27. Van Pottelberge GR, Mestdagh P, Bracke KR, et al. MicroRNA expression in induced sputum of smokers and patients with chronic obstructive pulmonary disease. Am J Respir Crit Care Med. 2011. 183(7): 898-906. doi: http://dx.doi.org/10.1164/rccm.201002-0304OC

28. D'Hulst A I, Bracke KR, Maes T, et al. Role of tumour necrosis factor-alpha receptor $\mathrm{p} 75$ in cigarette smoke-induced pulmonary inflammation and emphysema. Eur Respir J. 2006. 28(1): 102112. doi: http://dx.doi.org/10.1183/09031936.06.00059305

29. Hassan F, Nuovo GJ, Crawford M, et al. MiR-101 and miR-144 regulate the expression of the CFTR chloride channel in the lung. PLoS One. 2012. 7(11): e50837. doi: http://dx.doi.org/10.1371/journal.pone.0050837

30. Christenson SA, Brandsma CA, Campbell JD, et al. miR638 regulates gene expression networks associated with emphysematous lung destruction. Genome Med. 2013. 5(12): 114. doi: http://dx.doi.org/10.1186/gm519

31. Conrad KD, Niepmann M. The role of microRNAs in hepatitis C virus RNA replication. Arch Virol. 2014. 159(5): 849-862. doi: http://dx.doi.org/10.1007/s00705-013-1883-4

32. Janssen HL, Reesink HW, Lawitz EJ, et al. Treatment of HCV infection by targeting microRNA. $N$ Engl J Med. 2013. 368(18): 1685-1694. doi: http://dx.doi.org/10.1056/NEJMoa1209026

33. Kumar MS, Erkeland SJ, Pester RE, et al. Suppression of nonsmall cell lung tumor development by the let-7 microRNA family. Proc Natl Acad Sci U S A. 2008. 105(10): 3903-3908. doi: http://dx.doi.org/10.1073/pnas.0712321105

34. Trang P, Medina PP, Wiggins JF, et al. Regression of murine lung tumors by the let-7 microRNA. Oncogene. 2010. 29(11): 15801587. doi: http://dx.doi.org/10.1038/onc.2009.445

35. Oglesby IK, McElvaney NG, Greene CM. MicroRNAs in inflammatory lung disease--master regulators or target practice? Respir Res. 2010. 11: 148. doi: http://dx.doi.org/10.1186/1465-9921-11-148

36. Chernolovskaya EL, Zenkova MA. Chemical modification of siRNA. Curr Opin Mol Ther. 2010. 12(2): 158-167.

37. Fujita Y, Takeshita F, Mizutani T, et al. A novel platform to enable inhaled naked RNAi medicine for lung cancer. Sci Rep. 2013. 3: 3325. doi: http://dx.doi.org/10.1038/srep03325

38. Fujita Y, Takeshita F, Kuwano K, Ochiya T. RNAi Therapeutic Platforms for Lung Diseases. Pharmaceuticals (Basel). 2013. 6(2): 223-250. doi: http://dx.doi.org/10.3390/ph6020223

39. Fujita Y, Kosaka N, Araya J, Kuwano K, Ochiya T. Extracellular vesicles in lung microenvironment and pathogenesis. Trends Mol Med. 2015. doi: http://dx.doi.org/10.1016/j.molmed.2015.07.004

40. Ismail N, Wang Y, Dakhlallah D, et al. Macrophage microvesicles induce macrophage differentiation and miR-223 transfer. Blood. 2013. 121(6): 984-995. doi: http://dx.doi.org/10.1182/blood-2011-08-374793

41. Kulshreshtha A, Ahmad T, Agrawal A, Ghosh B. Proinflammatory role of epithelial cell-derived exosomes in allergic airway inflammation. J Allergy Clin Immunol. 2013. 131(4): 1194-1203. 203 e1-14. doi: http://dx.doi.org/10.1016/j.jaci.2012.12.1565

42. Fujita Y, Yoshioka Y, Ito S, et al. Intercellular communication by extracellular vesicles and their microRNAs in asthma. Clin Ther. 2014. 36(6): 873-881.

doi: http://dx.doi.org/10.1016/j.clinthera.2014.05.006
43. Takahashi T, Kubo H. The role of microparticles in chronic obstructive pulmonary disease. Int J Chron Obstruct Pulmon Dis. 2014. 9: 303-314.

doi: http://dx.doi.org/10.2147/COPD.S38931

44. Gordon C, Gudi K, Krause A, et al. Circulating endothelial microparticles as a measure of early lung destruction in cigarette smokers. Am J Respir Crit Care Med. 2011. 184(2): 224-232. doi: http://dx.doi.org/10.1164/rccm.201012-2061OC

45. Takahashi T, Kobayashi S, Fujino N, et al. Annual FEV 1 changes and numbers of circulating endothelial microparticles in patients with COPD: a prospective study. BMJ Open. 2014. 4(3): e004571.

doi: http://dx.doi.org/10.1136/bmjopen-2013-004571

46. Brosius J. Waste not, want not--transcript excess in multicellular eukaryotes. Trends Genet. 2005. 21(5): 287-288. doi: http://dx.doi.org/10.1016/j.tig.2005.02.014

47. Lee JT. Epigenetic regulation by long noncoding RNAs. Science. 2012. 338(6113): 1435-1439. doi: http://dx.doi.org/10.1126/science.1231776

48. Ji P, Diederichs $\mathrm{S}$, Wang W, et al. MALAT-1, a novel noncoding RNA, and thymosin beta4 predict metastasis and survival in early-stage non-small cell lung cancer. Oncogene. 2003. 22(39): 8031-8041. doi: http://dx.doi.org/10.1038/sj.onc.1206928

49. Nakagawa T, Endo H, Yokoyama $M$, et al. Large noncoding RNA HOTAIR enhances aggressive biological behavior and is associated with short disease-free survival in human non-small cell lung cancer. Biochem Biophys Res Commun. 2013. 436(2): 319-324. doi: http://dx.doi.org/10.1016/j.bbrc.2013.05.101

50. Thai P, Statt S, Chen $\mathrm{CH}$, et al. Characterization of a novel long noncoding RNA, SCAL1, induced by cigarette smoke and elevated in lung cancer cell lines. Am J Respir Cell Mol Biol. 2013. 49(2): 204-211. doi: http://dx.doi.org/10.1165/rcmb.2013-0159RC

51. Rangasamy T, Cho CY, Thimmulappa RK, et al. Genetic ablation of Nrf2 enhances susceptibility to cigarette smoke-induced emphysema in mice. J Clin Invest. 2004. 114(9): 1248-1259. doi: http://dx.doi.org/10.1172/JCI21146 